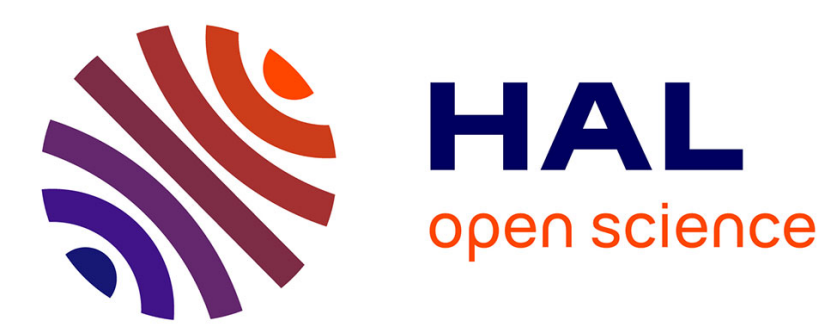

\title{
Decoupling Path Following and Velocity Profile in Vision-Guided Laser Steering.
}

Jean-Antoine Seon, Brahim Tamadazte, Nicolas Andreff

\section{To cite this version:}

Jean-Antoine Seon, Brahim Tamadazte, Nicolas Andreff. Decoupling Path Following and Velocity Profile in Vision-Guided Laser Steering.. IEEE Transactions on Robotics, 2015, 31 (2), pp.280-289. 10.1109/TRO.2015.2400660 . hal-01142126

\section{HAL Id: hal-01142126 \\ https://hal.science/hal-01142126}

Submitted on 14 Apr 2015

HAL is a multi-disciplinary open access archive for the deposit and dissemination of scientific research documents, whether they are published or not. The documents may come from teaching and research institutions in France or abroad, or from public or private research centers.
L'archive ouverte pluridisciplinaire HAL, est destinée au dépôt et à la diffusion de documents scientifiques de niveau recherche, publiés ou non, émanant des établissements d'enseignement et de recherche français ou étrangers, des laboratoires publics ou privés. 


\title{
Decoupling Path Following and Velocity Profile in Vision-guided Laser Steering
}

\author{
Jean-Antoine Seon, Brahim Tamadazte, and Nicolas Andreff
}

\begin{abstract}
Laser surgery requires accurate following of a path defined by the surgeon, while the velocity on this path is dependent on the laser-tissue interaction. Therefore, path following and velocity profile control must be decoupled. In this paper, non-holonomic control of the unicycle model is used to implement velocity-independent visual path following for laser surgery. The proposed controller was tested, in simulation as well as experimentally in several conditions of use: different initial velocities (step input, successive step inputs, sinusoidal inputs), optimized/non-optimized gains, time-varying path (simulating a patient breathing), and complex curves with curvatures. Thereby, experiments at $587 \mathrm{~Hz}$ (frames/second) show an average accuracy lower than 0.22 pixels $(\approx 10 \mu \mathrm{m})$ with a standard deviation of 0.55 pixels $(\approx 25 \mu \mathrm{m})$ path following, and a relative velocity distortion of less than $10^{-6} \%$.
\end{abstract}

Index Terms-Non-holonomic control, visual servoing, visionguided laser, laser surgery, endoluminal interventions, surgical robot, intracorporal micro-robotics.

\section{INTRODUCTION}

Laser surgery consists of the use of a surgical laser (instead of a scalpel) to cut tissue in which the laser beam vaporizes the cells. A large variety of surgical areas practice laser surgery: ophthalmology, gynaecology, otolaryngology, neurosurgery, and paediatric surgery [1]. This is especially true when it comes to microsurgery which requires an extreme precision [2].

For instance, vocal folds surgery is extremely demanding in terms of accuracy because of the specific tissue to be resected (thin, viscous, fragile, difficult healing, and lesions which can be less than $1 \mathrm{~mm}$ ) and the necessity to preserve the patient voice [3] [4].

Currently, the most successful protocol for vocal folds surgery, widely used in hospitals is certainly the suspension laryngoscopic technique which consists of a straight-rigid laryngoscope, a stereomicroscope, a set of specific and miniatures surgical instruments, a laser source, and a foot-pedal controller device to activate the laser [3]. In fact, the most popular microphonosurgery procedure uses the AcuBlade ${ }^{\circledR}$ system [5] which allows good diagnostics and microsurgeries procedures. However, this system suffers from several drawbacks, especially in terms of accuracy (laser source placed outside the patient at $400 \mathrm{~mm}$ from the vocal folds) and ease of operation (teleoperation mode only). Consequently, the laser

The authors are with FEMTO-ST Institute, AS2M department, Université de Franche-Comté/CNRS/ENSMM/UTBM, 24 rue Savary, 25000 Besanọn, France

Part of this work is presented at the 3rd Workshop on Visual Control of Mobile Robots (ViCoMor), IEEE/RSJ International Conference on Intelligent Robots and Systems (IROS), 2014

Manuscript received xx, 2014; revised xxx, 2014. surgery performances are highly dependent on the individual surgeon dexterity and skills [6].

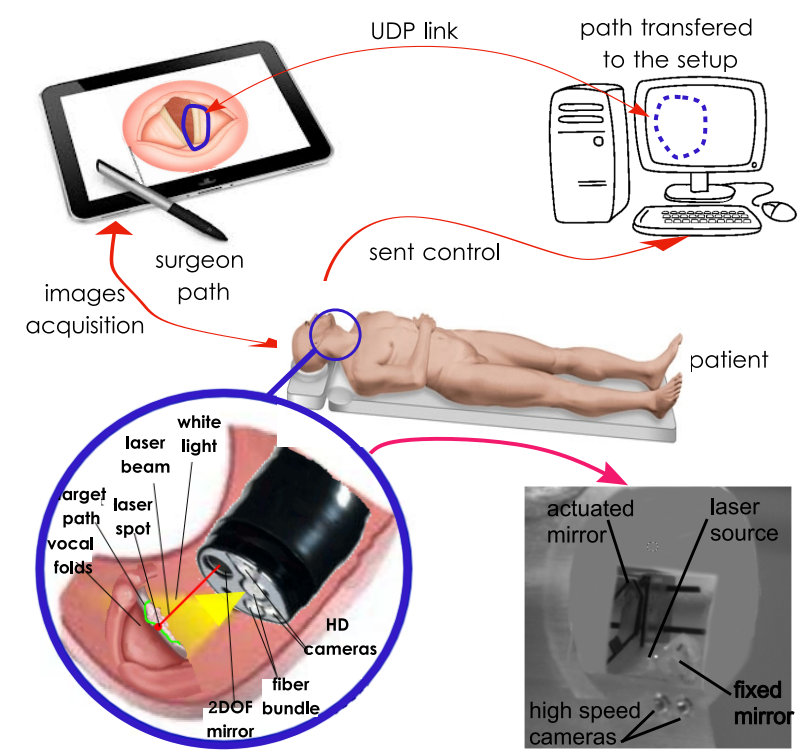

Fig. 1: Global view of the vocal folds surgery procedure.

Generally, in the case of laser surgeries, the control consists of two tasks. The first one is to ensure the laser velocity compatibility with laser-tissue interaction (longitudinal control), in order to avoid carbonization of the tissue while achieving efficient incision or ablation. The second one (lateral control) is to ensure that the laser follows the desired geometric path drawn by the surgeon on the input device (e.g., a tablet in [7]) (Fig. 1). These two tasks eventually define the trajectory (i.e., geometric path + velocity profile along the path) of the laser. However, it is not advisable to use standard trajectory tracking because the two tasks should be intuitively modifiable by the surgeon. In fact, the velocity of the laser displacements must be the same regardless of the shape, the size or the curvature of the incision path. In addition, the norm of this velocity must be adapted, by the physician, in function of the tissue to be resected (thin, thick, fragile, etc.) or the laser source (CO2 laser, Helium-neon laser, etc.). In this paper, we focus especially on the second task: laser path following using the visual feedback independently from the velocity profile.

Moreover, beyond laser surgery applications, efficient path following in which the velocity profile (easily tuned) is invariant to the path (non-parametrized curve) size, shape, curvature, sampling period, ... can be an important issue for industrial applications that use laser scanning process. There are several works reported in the literature, especially in visionbased control supervision of welding process [8] or trajectory 
tracking (with low curvature) using a robotic arm which embeds the laser source [9], [10], [11]. In addition, regular and precise path following could find applications in 2D/3D laser micromachining (e.g., microsystems fabrication) [14] as well as in free-contact micromanipulation techniques (e.g., laser trapping) [15]. Yet, as far as we could understand it, all those work use trajectory tracking instead of path following, making the longitudinal controller blind to variations in laser-matter interaction.

The first contribution of the paper is to consider, on purpose, laser path following as a non-holonomic problem, similar to unicycle path following. The second contribution of the paper is to implement path following at high frequency to satisfy the constraints of laser-tissue interaction.

In the remainder of this paper, Section II relates laser path following in the image and unicycle path following on the ground. In Section III a background on the existing path following methods is given while Section IV deals with the implementation for laser path following technique. Section V presents simulation results as well as experimental validation.

\section{LASER PATH FOLLOWING IN THE IMAGE $v s$. UNICYCLE PATH FOLLOWING ON THE GROUND}

Let us first show that laser path following in the image is very similar to unicycle path following on the ground (represented by the block diagram shown in Fig. 2)

- in both cases, the direction and the amplitude of the velocity are servoed independently;

- in the unicycle case, the velocity is generally expected to be such that the curvilinear velocity is constant while in the case of laser surgery, the velocity is expected to match a constant velocity on the tissue surface;

- in both cases, the path following accuracy is expected to be independent from the velocity amplitude.

- both laser spot and unicycle velocities should be smooth.

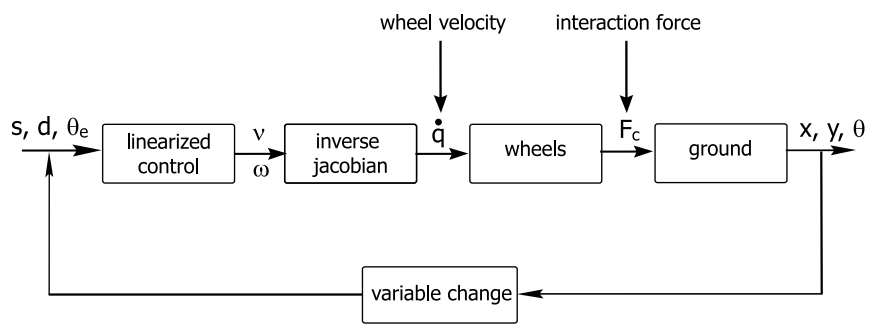

Fig. 2: Block diagram for the unicycle kinematic model.

With respect to these similarities, we thus chose to implement the unicycle model for the path following in the image. Indeed, imposing the unicycle non-holonomic constraint on the holonomic laser manipulator as shown in Fig. 3 which represents the laser steering kinematic model developed in this paper. This allows to

- ensure smoothness of the laser motion which is a key issue in laser surgery quality;

- control the laser displacement with a uniform velocity along the path;

- exploit efficient path following algorithms.
These similarities and differences between the path following in the image and the path following in the ground can be perfectly illustrated by the schemes given in Fig. 4. Namely,

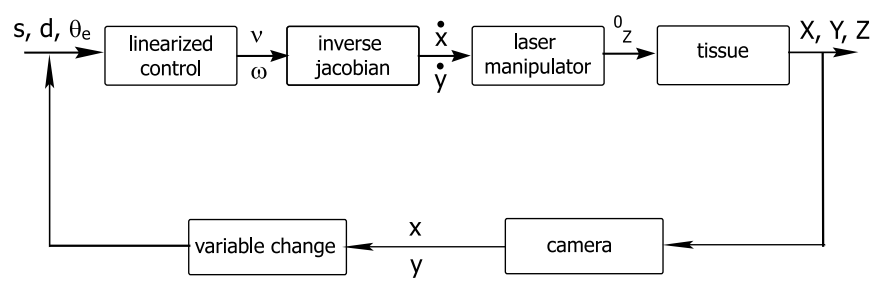

Fig. 3: Block diagram for the laser steering kinematic model.

we want to control, as for a mobile robot, the lateral derivation to the path $(d)$ and the orientation error $\left(\theta_{e}\right)$ between the tangent vector to the path and the robot velocity direction to achieve a smooth motion.

\section{PATH FOLLOWING}

\section{A. State of the Art}

Path following in mobile robotics has been widely studied in the literature for many years and has found several applications in the industrial field (e.g., autonomous vehicle control [13], farming application [12]). Path following differs from trajectory following essentially by the fact that the notion of time is removed in the first one. Indeed, in trajectory following the robot is controlled to be at a location at a given time which is not the case in path following. Indeed, as shown by Brockett [16], mobile robots are not stable with continuous steady state feedback laws. The first alternative approach is to use discontinuous control laws [17] or piecewise continuous control law [18], but these methods do not explicitly address the issue of robustness to the occurrence of uncertainties. Thereafter, [19] proposed to use a sliding mode based controller which has shown better behaviors. The second approach is to use continuous non-steady state feedback laws such as Samson in the early 90 [20]. Lyapunov methods are often used to design such type of control law [21], [22]. Indeed, it gives good results in terms of robustness and accuracy. It is also possible to use a chained system to design the controller with exact linearization as shown by Morin and Samson [23], [24]. In these studies, the path following speed profile is not known. So, only the path and the distance between the robot and the curve are considered. Therefore, the tangential vectors of the curve are used to set the velocity and the orientation of the robot.

\section{B. Notations}

For a better understanding of the different notations introduced in this paper, the reader may refer to the following Table 1 .

\section{Kinematic Equations}

Let us consider the mobile robot (laser spot) represented by a $2 \mathrm{D}$ point $\mathbf{p}=(x, y)^{\top}$ in a fixed (reference) frame $\mathbf{R}_{\mathbf{0}}$ (the 


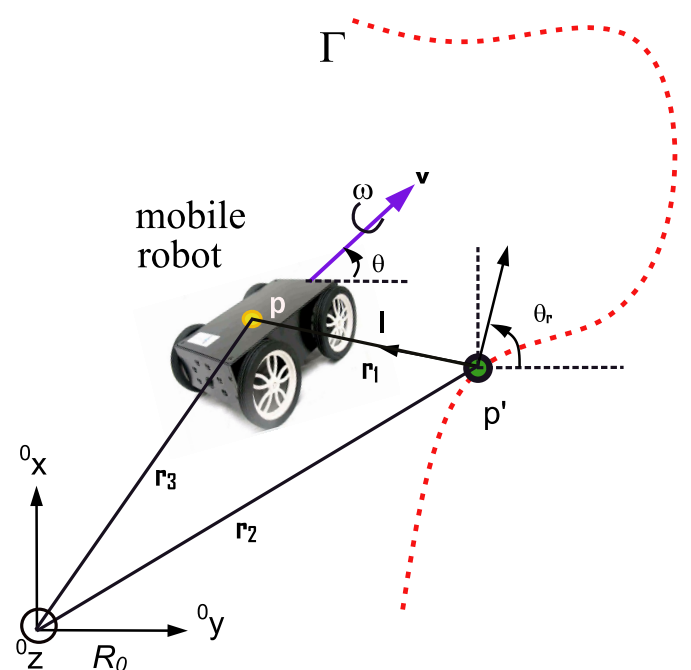

(a)

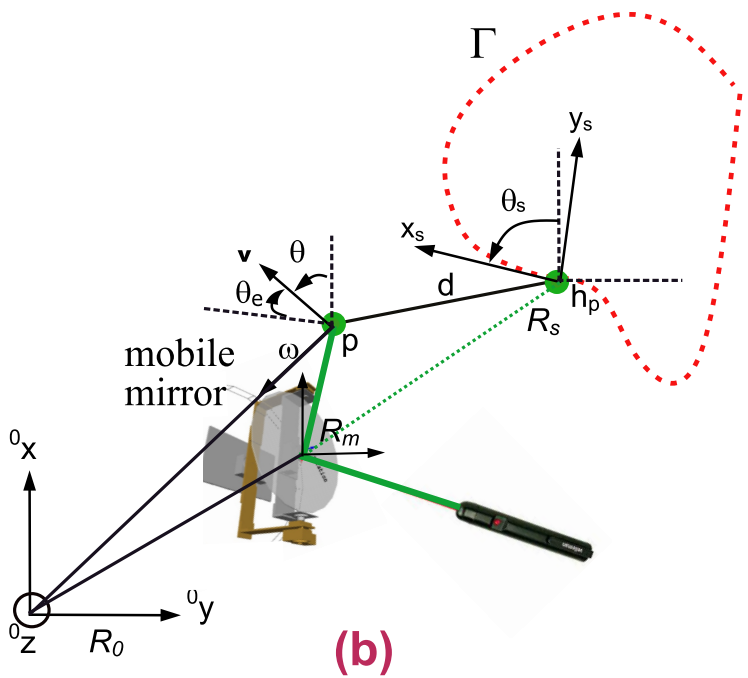

Fig. 4: Similarities between unicycle vehicle kinematic model (a) and a laser steering kinematic model (b).

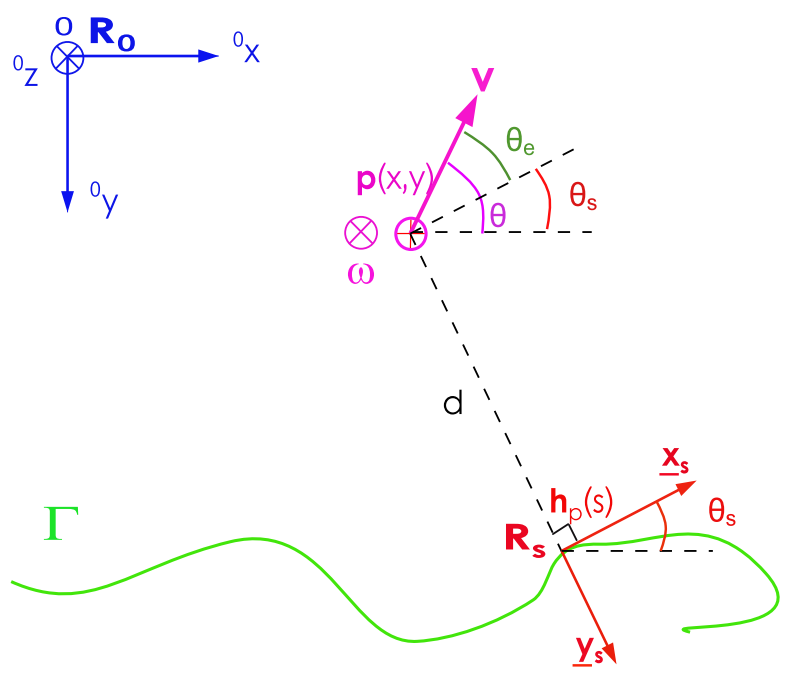

Fig. 5: Representation of the different frames used in the design of the path following method.

\begin{tabular}{|c|c|}
\hline notations & description \\
\hline$\Gamma$ & path (curve) to be followed by the laser spot \\
\hline C & set of points that approximate $\Gamma$ \\
\hline $\mathbf{c}_{j}$ & $j^{\text {th }}$ point of the set $\mathcal{C}$ \\
\hline $\mathbf{R}_{\mathbf{o}}$ and $\mathbf{R}_{\mathbf{s}}$ & image frame and Frenet frame, resp. \\
\hline$x, y$ and $z$ & unit vectors of $\mathbf{R}_{\mathbf{o}}$ \\
\hline$\underline{\mathbf{x}}_{s}, \underline{\mathbf{y}}_{s}$ and $\underline{\mathbf{z}}_{s}$ & unit vectors of $\mathbf{R}_{\mathbf{s}}$ \\
\hline $\mathbf{p}=(x, y)^{\top}$ & 2D point (laser spot) represented in $\mathbf{R}_{\mathbf{o}}$ \\
\hline$\dot{x}$, and $\dot{y}$ & $\begin{array}{l}\text { laser spot velocities in the image along } \\
{ }^{0} x,{ }^{0} y \text {, resp. }\end{array}$ \\
\hline$\theta$ & angle between the laser velocity direction $\underline{\mathbf{v}}$ and ${ }^{0} x$ \\
\hline$\theta_{e}$ and $\dot{\theta_{e}}$ & $\begin{array}{l}\text { orientation error of the laser spot with respect to } C \\
\text { and its time-variation, resp. }\end{array}$ \\
\hline$v$ & velocity amplitude of the laser spot in $\mathbf{R}_{\mathbf{s}}$ \\
\hline$v_{a c h}$ & achieved velocity of the laser spot in $\mathbf{R}_{\mathbf{s}}$ \\
\hline$\underline{\mathbf{v}}_{k}$ & velocity direction of the laser spot at each iteration $k$ \\
\hline & velocity (amplitude and direction) of the spot \\
\hline $\mathbf{h}_{p}=\left(x_{\mathbf{h}_{p}}, y_{\mathbf{h}_{p}}\right)^{\top}$ & orthogonal projection of $\mathbf{p}(x, y)^{\top}$ on $\mathcal{C}$ \\
\hline & rotational velocity carried by the axis ${ }^{0} z$ \\
\hline $\begin{array}{l}s \text { and } \dot{s} \\
s\left(\mathbf{c}_{j}\right) \text { and } s\left(\mathbf{h}_{p}\right)\end{array}$ & $\begin{array}{l}\text { curvilinear abscissa and its time-variation, resp. } \\
\text { curvilinear abscissa on the point } \mathbf{c}_{j} \text { and } \mathbf{h}_{p}\end{array}$ \\
\hline$C(s)$ and $\frac{\partial C(s)}{\partial s}$ & $\begin{array}{l}\text { curve curvature at the abscissa } s \\
\text { and its curvilinear abscissa variation }\end{array}$ \\
\hline$d$ and $d$ & distance of $\mathbf{p}(x, y)$ to $C$ and its time-variation \\
\hline$z_{1}, z_{2}$ and $z_{3}$ & $\begin{array}{l}\text { variables used in the chained equations system } \\
\text { (local transformation of } u_{1}, u_{2} \text { ) }\end{array}$ \\
\hline$u_{1}$ and $u_{2}$ & intermidate control inputs in a local transformation \\
\hline $\begin{array}{l}\gamma_{1} \text { and } \gamma_{2} \\
T_{e}\end{array}$ & $\begin{array}{l}\text { positive gains for the laser spot velocity tuning } \\
\text { sampling period }\end{array}$ \\
\hline$\widehat{s_{k+T_{e}}}$ & $\begin{array}{l}\text { predicted curvilinear abscissa at each iteration } k \\
\text { inverse differential kinematic matrix of the } \\
\text { laser manipulator }\end{array}$ \\
\hline$\dot{\mathbf{q}}_{i}$ & joint velocity of each joint $i$ of the manipulator \\
\hline$r$ & weighing coefficient for the line $\left[\mathbf{c}_{j-1} ; \mathbf{c}_{j+1}\right]$ \\
\hline
\end{tabular}

TABLE I: Notations used in this paper.

image frame). The kinematics of the unicycle are governed in the world frame by

$$
\begin{aligned}
\dot{x} & =v \cos \theta \\
\dot{y} & =v \sin \theta \\
\dot{\theta} & =\omega
\end{aligned}
$$

where $\theta$ is the angle between laser velocity direction $\underline{\mathbf{v}}$ and the ${ }^{0} x$ axis of the world frame, $v$ represents the translational velocity amplitude of the laser spot in $\mathbf{R}_{\mathbf{s}}\left(\mathbf{v}=v \underline{\mathbf{v}}_{k}\right), \mathbf{h}_{p}(s)$ is the orthogonal projection of $\mathbf{p}=(x, y)^{\top}$ on $\Gamma$ and $\omega$ its rotational velocity carried by the axis ${ }^{0} z$ (Fig. 5).

These equations can be generalized in the Frenet frame $\mathbf{R}_{\mathbf{s}}$ attached to the path (Fig. 5) as shown by Morin and Samson [23], [24]. Consequently, the kinematics become governed in $\mathbf{R}_{\mathbf{s}}$ by

$$
\begin{aligned}
\dot{s} & =\frac{v}{1-d C(s)} \cos \theta_{e} \\
\dot{d} & =v \sin \theta_{e} \\
\dot{\theta}_{e} & =\omega-\dot{s} C(s)
\end{aligned}
$$

where $s$ and $C(s)$ are respectively the curvilinear abscissa and the curvature, $\theta_{e}$ is the difference between the laser orientation $\underline{\mathbf{v}}$ and the tangential vector of $\mathbf{R}_{\mathbf{s}}$ and $d$ is the shortest distance of $\mathbf{p}=(x, y)^{\top}$ to $\Gamma$.

\section{Control law}

To establish the controller, [23] introduced a coordinates/variables transformation in the following manner: 
$\left\{s, d, \theta_{e}, v, \omega\right\} \Longleftrightarrow\left\{z_{1}, z_{2}, z_{3}, u_{1}, u_{2}\right\}$ defined in $\mathbb{R}^{2} \times(-$ $\left.\frac{\pi}{2},+\frac{\pi}{2}\right) \times \mathbb{R}^{2}$. This allows to transform locally (4), (5) and (6) in

$$
\begin{aligned}
& \dot{z}_{1}=u_{1} \\
& \dot{z}_{2}=u_{1} z_{3} \\
& \dot{z}_{3}=u_{2}
\end{aligned}
$$

where $u_{1}$ and $u_{2}$ are intermediate control inputs.

Thereby, if $z_{1}=s$, then (7) implies

$$
u_{1}=\frac{v}{1-d C(s)} \cos \theta_{e}
$$

In the same way if $z_{2}=d$, then (8) implies

$$
z_{3}=(1-d C(s)) \tan \theta_{e}
$$

As a consequence and using (9), $u_{2}$ is defined as

$$
\begin{array}{r}
u_{2}=\left(-\dot{d} C(s)-d \frac{\partial C(s)}{\partial s} \dot{s}\right) \tan \theta_{e} \\
+(1-d C(s))\left(1+\tan ^{2} \theta_{e}\right)(\omega-\dot{s} C(s))
\end{array}
$$

Finally, to ensure that the distance $d$ and the orientation error $\theta_{e}$ are servoed to 0 , the stable proportional state feedback solution [23] is

$$
u_{2}=-u_{1} \gamma_{1} z_{2}-\left|u_{1}\right| \gamma_{2} z_{3}
$$

where $\gamma_{1}$ and $\gamma_{2}$ are positive gains and $u_{1} \neq 0$ can be chosen arbitrarily.

\section{E. Stability Conditions}

Therefore, the controller is asymptotically stable when $u_{1}=$ $\dot{s}$ is constant (positive or negative). However, the system is not controlled directly by $u_{1}$ but by $v$. So, a stability condition on $v$ is needed. It has been shown in [24] that the stability is ensured with

- $v$ must be a bounded differentiable time-function;

- $v$ must not tend to zero when $t$ tends to infinity;

- $\dot{v}$ must be bounded.

\section{IMPLEMENTATION}

\section{A. Laser Surgery Considerations}

Up to now, the parameters of the curve $\Gamma$ are considered as perfectly known as in the case of the path planning for mobile robotics. Remind that in laser surgery, the controller receives the normalized and sampled coordinates of the path while the surgeon draws $\Gamma$ (using a tablet) as illustrated in Fig. 1. Therefore, the path used to control the laser displacements is an approximation (yet very accurate) of the real curve drawn by the surgeon.

As the curvature $C(s)$ needs to be computed for the control law, the parameterized path has to be at least $C^{2}(C(s)$ is the time-derivation of the tangent vector). However, the fact that the path is a non-parameterized curve, then the curvature and the radius at each point are computed using 3 successive points. Obviously the quality of this approximation and the smoothness of the path depend on the number of points.
Consider that the path $\Gamma$ is described by a set of points $C=$ $\left\{\mathbf{c}_{i}, i=1 \ldots n\right\}$ with the relation $\mathbf{c}_{1}<\mathbf{c}_{2}<\ldots<\mathbf{c}_{n}$. Each $\mathbf{c}_{i}$ is a quadruplet containing two Cartesian coordinates $(x, y)$ and two curvilinear coordinates (curvature $C(s)$ and curvilinear abscissa $s$ ).

In order to control the laser spot displacements with regards to the path drawn by the surgeon, the following methodology (Algorithm 1) has been developed, implemented and validated in simulation and experimental set-up.

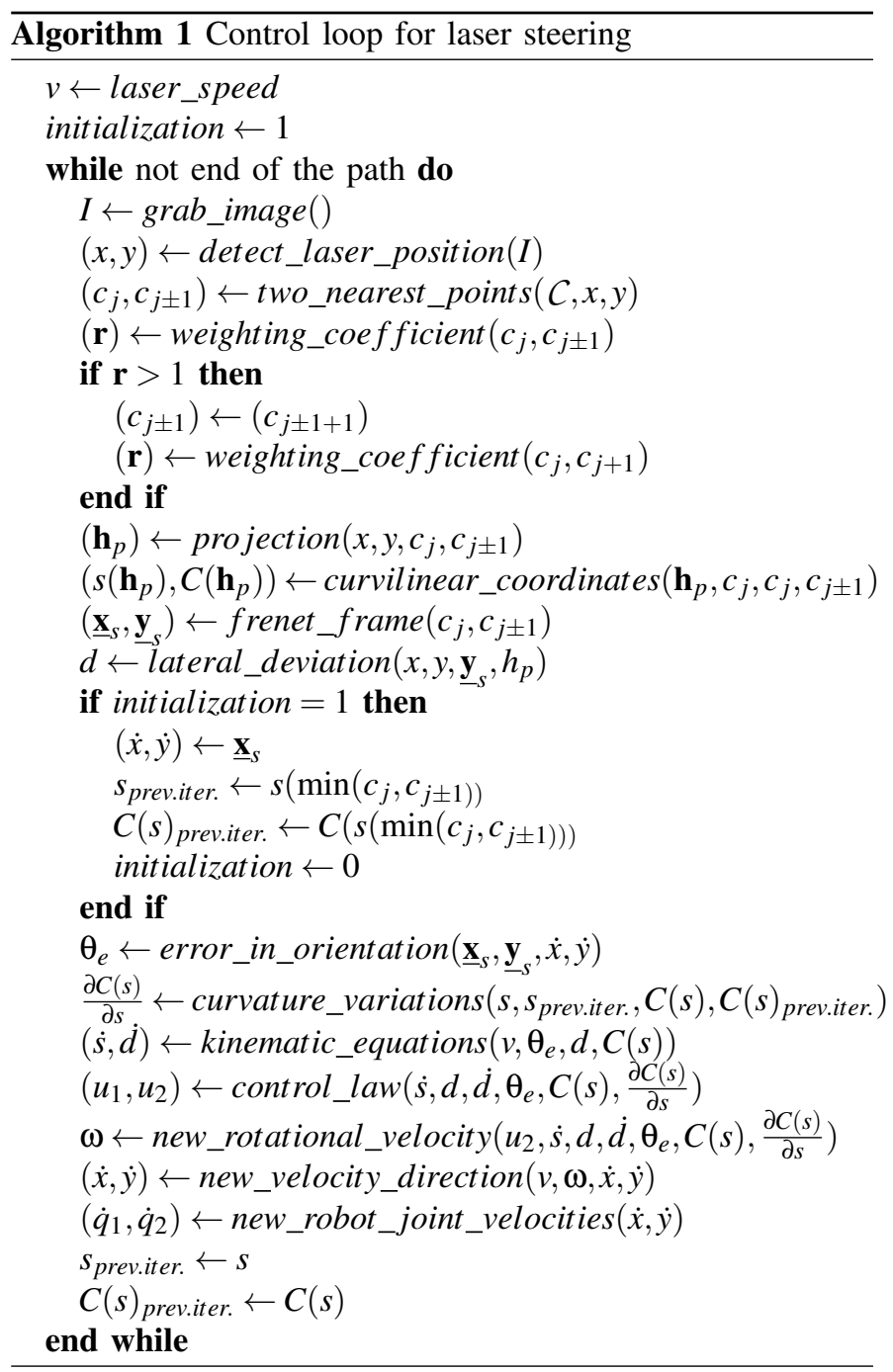

\section{B. Computation of the closest point on the path}

The position of the laser spot $\mathbf{p}_{k}=\left(x_{k}, y_{k}\right)^{\top}$ is known at each iteration $k$. Thus, it is possible to identify both the closest two successive points in $\mathcal{C}$, i.e., $\mathbf{c}_{j}$ and $\mathbf{c}_{j \pm 1}$ (with $d\left(\mathbf{c}_{j}\right)<$ $\left.d\left(\mathbf{c}_{j \pm 1}\right)\right)$ as shown by Fig. 6. To do so, we perform a local search around the predicted curvilinear abscissa $s_{k}$ given by

$$
\widehat{s_{k+T_{e}}}=\dot{s} T_{e}+s_{k}
$$

with $T_{e}$ the sampling period on $\Gamma$. This allow to avoid searching through all the curve and be caught in ambiguities when, for instance, the path cross itself. Also, using $\mathbf{c}_{j}$ and $\mathbf{c}_{j \pm 1}$ allows to compute the line projection of $\mathbf{p}$ on the approximated 


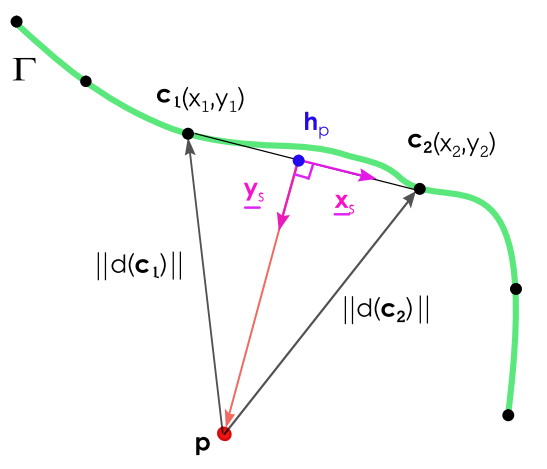

Fig. 6: Estimation of the distance $d$ at each iteration $k$.

path $\mathcal{C}$ which gives $\mathbf{h}_{p}=\left(x_{\mathbf{h}_{p}}, y_{\mathbf{h}_{p}}\right)^{\top}$. As shown in Fig. 6, the coordinates $x_{\mathbf{h}_{p}}$ and $y_{\mathbf{h}_{p}}$ can be computed by

$$
\begin{aligned}
& x_{\mathbf{h}_{p}}=x_{\min \left(\mathbf{c}_{j}, \mathbf{c}_{j \pm 1}\right)}+\mathbf{r}\left(x_{\max \left(\mathbf{c}_{j}, \mathbf{c}_{j \pm 1}\right)}-x_{\min \left(\mathbf{c}_{j}, \mathbf{c}_{j \pm 1}\right)}\right) \\
& y_{\mathbf{h}_{p}}=y_{\min \left(\mathbf{c}_{j}, \mathbf{c}_{j \pm 1}\right)}+\mathbf{r}\left(y_{\max \left(\mathbf{c}_{j}, \mathbf{c}_{j \pm 1}\right)}-y_{\min \left(\mathbf{c}_{j}, \mathbf{c}_{j \pm 1}\right)}\right)
\end{aligned}
$$

where $\mathbf{r}$ is the weighting coefficient on the segment $\left[\mathbf{c}_{j}, \mathbf{c}_{j \pm 1}\right]$ which can be computed using

$$
\begin{aligned}
\mathbf{r} & =\frac{\left(x_{\min \left(\mathbf{c}_{j}, \mathbf{c}_{j \pm 1}\right)}-x_{\max \left(\mathbf{c}_{j}, \mathbf{c}_{j \pm 1}\right)}\right)\left(x_{\min \left(\mathbf{c}_{j}, \mathbf{c}_{j \pm 1}\right)}-x_{k}\right)}{L^{2}} \\
& +\frac{\left(y_{\min \left(\mathbf{c}_{j}, \mathbf{c}_{j \pm 1}\right)}-y_{\max \left(\mathbf{c}_{j}, \mathbf{c}_{j \pm 1}\right)}\right)\left(y_{\min \left(\mathbf{c}_{j}, \mathbf{c}_{j \pm 1}\right)}-y_{k}\right)}{L^{2}}
\end{aligned}
$$

where $L=\left(\left(x_{\mathbf{c}_{j}}-x_{\mathbf{c}_{j \pm 1}}\right)^{2}+\left(y_{\mathbf{c}_{j}}-y_{\mathbf{c}_{j \pm 1}}\right)^{2}\right)^{\frac{1}{2}}$.

$\mathbf{r}$ is also useful in case where the points of $\mathcal{C}$ are not homogeneously distributed. Indeed, if $\mathbf{r}>1$ then the projection of the laser spot will be outside $\Gamma$ (the two closest points are not the two points describing the segment on which the laser spot should be located).

\section{Computation of the laser state in the Frenet frame}

Firstly, the curvilinear coordinates of the point $\mathbf{h}_{p}$ can be easily computed. Thus, the curvilinear abscissa $s\left(\mathbf{h}_{p}\right)$ is computed as the addition of the curvilinear abscissa of $\min \left(\mathbf{c}_{j}, \mathbf{c}_{j \pm 1}\right)$ and the distance between this point and $\mathbf{h}_{p}$, and the curvature $C(s)$ in $\mathbf{h}_{p}$ (i.e., $C\left(\mathbf{h}_{p}\right)$ ) is computed as follows

$$
\begin{aligned}
C\left(\mathbf{h}_{p}\right) & =C\left(s\left(\min \left(\mathbf{c}_{j}, \mathbf{c}_{j \pm 1}\right)\right)\right)(1-\mathbf{r}) \\
& +C\left(s\left(\max \left(\mathbf{c}_{j}, \mathbf{c}_{j \pm 1}\right)\right)\right) \mathbf{r}
\end{aligned}
$$

Moreover, the unit-vectors $\underline{\mathbf{x}}_{s}$ and $\underline{\mathbf{y}}_{s}$ defining the Frenet frame $\mathbf{R}_{\mathbf{s}}$ are determined using the line between $\mathbf{c}_{j}$ and $\mathbf{c}_{j \pm 1}$ as shown in Fig. 6. Furthermore, $d$ is recomputed using

$$
d=\left(\mathbf{p}_{k}-\mathbf{h}_{p}\right) \cdot \underline{\mathbf{y}}_{s}
$$

where, $\left(\mathbf{p}_{k}-\mathbf{h}_{p}\right)$ is the normal vector along $\underline{\mathbf{y}}_{s}$ from the closest point to the laser spot position (Fig. 6) and the dot product (.) allows knowing the exact sign of the laser spot position relative to the curve $\Gamma$. As the Frenet frame $\mathbf{R}_{\mathbf{s}}$ is constructed directly on $\mathbf{h}_{p}$, it ensure that the two vectors $\left(\left(\mathbf{p}_{k}-\mathbf{h}_{p}\right)\right.$ and $\underline{\mathbf{y}}_{s}$ ) are always collinear.

Therefore, the orientation error $\theta_{e}$ at each iteration $k$ can be updated by

$$
\theta_{e}=\arctan 2\left(\underline{\mathbf{v}}_{k} \cdot \underline{\mathbf{x}}_{s}, \underline{\mathbf{v}}_{k} \cdot \underline{\mathbf{y}}_{s}\right)
$$

where $\mathbf{v}_{k}$ is the current velocity direction of the laser spot in the $\mathbf{R}_{\mathbf{0}}$ frame.

\section{Initialization}

As shown in (20), $\mathbf{v}_{k}$ is needed to compute the angle values at each iteration $k$. To do this, initialization values are fixed in order that the laser spot follows immediately the direction of the path. Thereby, the initial velocity of the laser spot is given by

$$
\mathbf{v}_{0}=v \underline{\mathbf{x}}_{s}
$$

\section{E. Constant velocity laser steering}

By knowing the laser spot state in the Frenet frame $\mathbf{R}_{\mathbf{s}}$ and by inverting (12), it is possible to define the new expression of $\omega$

$$
\omega=\frac{u_{2}+\left(\dot{d} C(s)+d \frac{\partial C(s)}{\partial s} \dot{s}\right) \tan \theta_{e}}{(1-d C(s))\left(1+\tan ^{2} \theta_{e}\right)}+\dot{s} C(s)
$$

From the latter, we derive the new expression of the laser spot velocity direction in the image by

$$
\underline{\mathbf{v}}_{k+1}=\frac{\underline{\mathbf{v}}_{k}+\omega^{0} \underline{\mathbf{z}} \times \underline{\mathbf{v}}_{k}}{\left\|\underline{\mathbf{v}}_{k}+\omega^{0} \underline{\mathbf{z}} \times \underline{\mathbf{v}}_{k}\right\|}
$$

where, $\times$ defines the cross-product and $\underline{\mathbf{v}}_{k}$ represents the current velocity direction of the laser spot.

Thereafter, it is necessary to convert these velocities to the joint velocities $\dot{\mathbf{q}}_{i}(i \in[1,2])$ through the inverse differential kinematic matrix $\mathbf{D}_{i n v}$ of the robot holding the laser as $\dot{\mathbf{q}}_{i}=$ $\mathbf{D}_{i n v} \underline{\mathbf{v}}_{k+1}$.

\section{VALIDATION}

\section{A. Experimental Set-up}

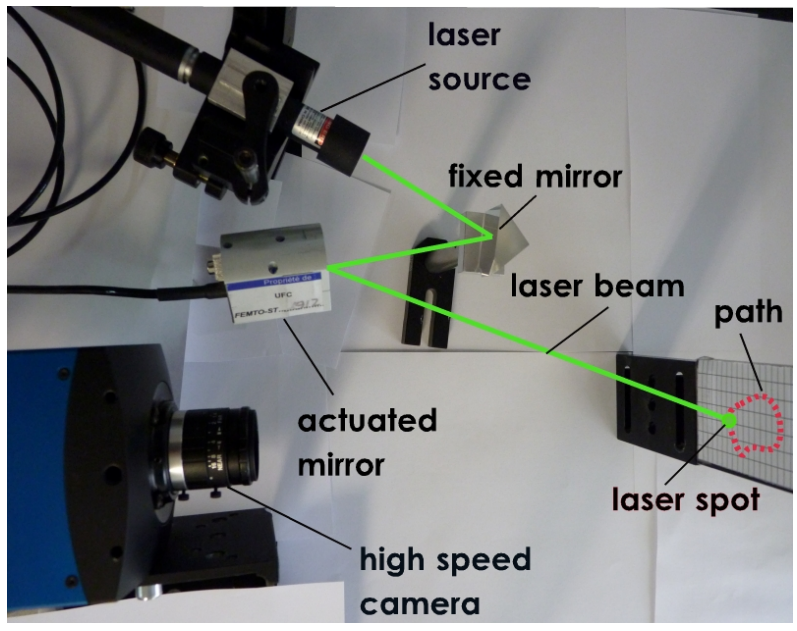

Fig. 7: Photography of the experimental set-up. 
The proposed path following method was tested on an experimental set-up (Fig. 7) which consists of a high speed camera EoSens ${ }^{\circledR}$ CXP (from Mikrotron ${ }^{\circledR}$ ) characterized with a frame rate which can reach 10000 frames per second (fps) with a resolution of $800 \times 600$ pixels, a laser source (i.e., a red laser pointer), a fixed mirror, and an actuated mirror (S-334) from Physical Instruments Inc characterized by a bandwidth of $1 \mathrm{kHz}$, a resolution of $0.2 \mu \mathrm{rad}$ and a motion range of $\pm 25 \mathrm{mrad}$. The latter contains two single axis units (i.e., $\alpha$ and $\beta$ ) working in parallel with one common pivot point. With that motion range and appropriate alignment of the mirror with the image, the inverse kinematics can be approximated by: $\mathbf{D}_{i n v} \approx\left(\begin{array}{ll}1 & 0 \\ 0 & 1\end{array}\right)$.

Due to the low motion range of the PI mirror (i.e., $\equiv 3^{\circ}$ ), it is impossible to scan a region of interest of $20 \mathrm{~mm} \times 20 \mathrm{~mm}$ which represent the average surface of vocal folds of an adult male (a little bit smaller for a female subject). It is for this reason that the target surface is positioned at a distance of $200 \mathrm{~mm}$ of the actuated mirror. However, we are working on the development of a large motion micromirror which will able to gives laser beam rotations of $43^{\circ}$ in each dof.

Concerning the time-varying position $\mathbf{p}$ of the laser spot in the image, it is tracked using ViSP library [25]. The tracking method is based on the computation of the gravity center (barycenter) of the laser spot in the image, i.e, a subpixel detection (in laser surgery, the laser spot has a size (diameter) of about $200 \mu \mathrm{m})$.

\section{B. Simulation Validation}

The proposed method is first validated in simulation in order to verify the relevance of the proposed path following scheme and define optimally some initial parameters of the technique (such $v, \gamma_{1}$ and $\gamma_{2}$ ) in different cases of use (shape and size of the curve, velocity profile, sampling period, simulation of the tissue interaction, etc.).

1) Example of a Simulation: To illustrate the simulation results, we opted for a non-parametric and arbitrary shape curve as shown in Fig. 8(a). In order to illustrate the relevance of the proposed approach, the initial parameters (gains) are tuned to get a laser velocity $v=20$ pixels/second, $\gamma_{1}=0.2$, and $\gamma_{2}=0.8$.

In this example, the initial position of the laser spot is placed with an initial orientation error $\theta_{e}=0 \mathrm{rad}$ and an initial distance $d=-15$ pixels. The laser spot is deliberately placed away from the curve in order to test how the system will respond to large error. The simulation results are given in Fig. 8 where, Fig. 8(a) shows the evolution of the laser spot position $\mathbf{p}$ relatively to the reference curve $\Gamma$ and Fig. 8(b) and (c) illustrate the evolution of the distance error $d$ and the orientation error $\theta_{e}$, respectively, versus number of iterations $k$. It can be noted that once the laser spot joined the curve $\Gamma$, $d$ and $\theta_{e}$ are maintained at 0 .

To estimate the accuracy of the proposed method, we compute the RMS error and the standard deviation (STD) in $d$ and $\theta_{e}$. It gives the following values: $\operatorname{RMS}(d)=0.1466$ pixels, $\operatorname{STD}(d)=0.1253$ pixels, $\operatorname{RMS}\left(\theta_{e}\right)=0.2138 \mathrm{rad}$, and $\operatorname{STD}\left(\theta_{e}\right)$ $=0.2138 \mathrm{rad}$.

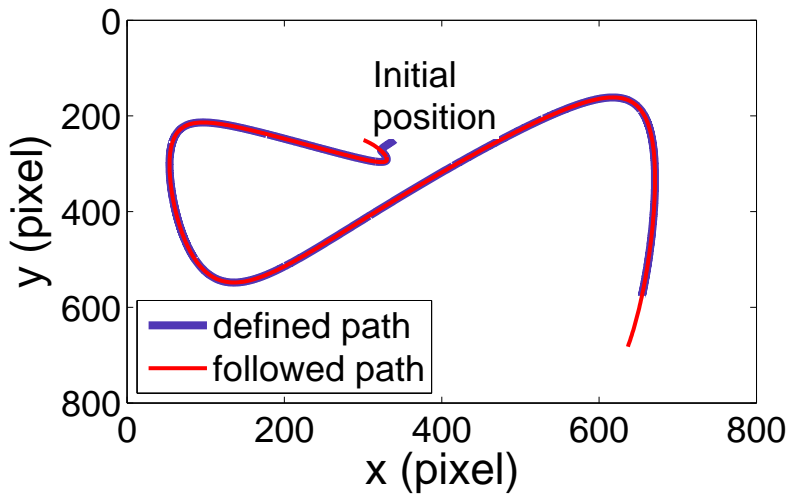

(a) Path following simulation.
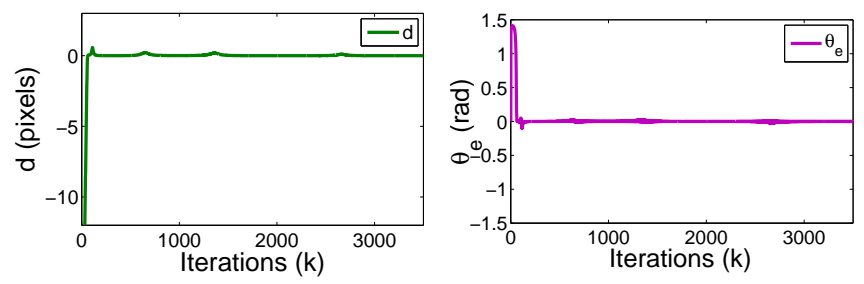

(b) Distance error $d$ and (c) orientation error $\theta_{e}$.

Fig. 8: Simulation results: (a) represents the path following achievement, (b) and (c) show the evolution of $\theta_{e}$ and $d$, respectively.
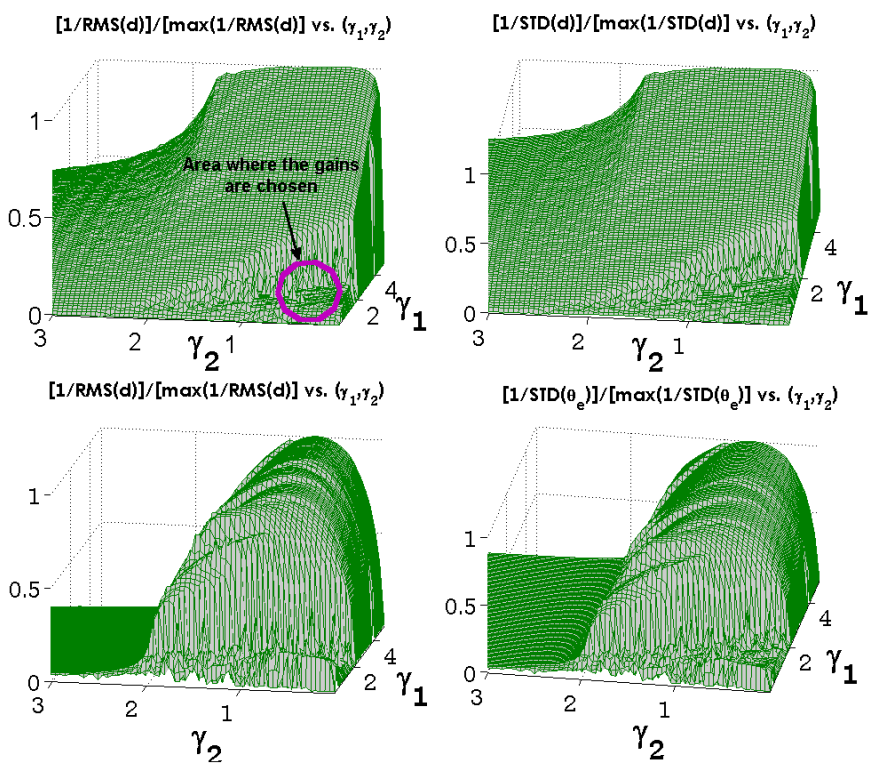

Fig. 9: Study of the influence of the gains $\gamma_{1}$ and $\gamma_{2}$ in the errors in $d$ and $\theta_{e}$.

2) Gains Tuning: In order to determine the best couple of gains for the controller, several simulations are performed using the same curve $\Gamma$ (Fig. 8) with varying $\gamma_{1}$ and $\gamma_{2}$. Then the accuracy of each path following simulation is compared using four criteria: RMS of the error in distance along the path $d$ and its STD, and the RMS of the orientation error $\theta_{e}$ and its STD (Fig. 9). This curve alternates between areas of high and low curvature but also between different directions so it is a good template for estimating the best parameters. 
The gains used for these simulations are included in the range $[0.01,5]$ with a step of 0.005 of each gain. The results are shown in Fig.9 where the closest values to 1 represent the best results. It can be highlighted that the error in distance along the path can be improved just by increasing the value of $\gamma_{1}$. This is directly related to the equation (13) where $\gamma_{1}$ controls the variable $z_{2}$ which represents $d$. In the same manner, increasing $\gamma_{2}$ will reduce the orientation error $\theta_{e}\left(\gamma_{2}\right.$ is related to $\tan \theta_{e}$ as shown in (11) and (13)). However, increasing significantly $\gamma_{2}$ induce a decrease in both distance and orientation errors because $\gamma_{2}$ is not depends only to $\theta_{e}$ but can interfere with $\gamma_{1}$, which may cause a disturbance in the controller.

With these simulations, the computed optimal gains on the range $[0.01,5]$ are located in $\gamma_{1}=5, \gamma_{2}=0.71$ considering that both $\theta_{e}$ and $d$ errors have the same impact on the quality of the path following. This set of gains gives the following errors: $\operatorname{RMS}(d)=0.0116$ pixels, $\operatorname{STD}(d)=0.0106$ pixels, $\operatorname{RMS}\left(\theta_{e}\right)=$ $0.0169 \mathrm{rad}, \operatorname{STD}\left(\theta_{e}\right)=0.0169 \mathrm{rad}$. However, even with nonoptimized parameters (example shown above), the proposed controller remains accurate, reliable and stable.

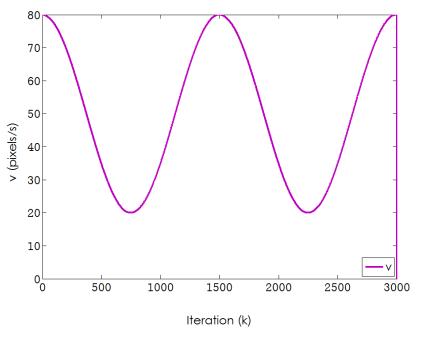

(a) sinusoidal inputs

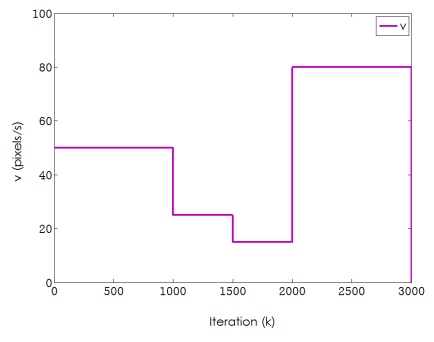

(b) steps inputs
Fig. 10: Various velocity profiles inputs during the path following process. (a) successiv step inputs and (b) sinusoid inputs.

3) Impact of $v$ during the Path Following Achievement: As mentioned above, in laser surgery, the velocity of the laser spot in the tissue needs to be adapted with respect to the tissue surface, heat profile, depth of lesion, etc. Therefore, in order to illustrate that the developed controller can be used in such application, a simulation has been performed in an unknown shape path following with arbitrary change of the velocity $v$ during the path following process. To do that, two velocity profiles are used: the first is with a smooth variation (sinusoidal profile) during the process (Fig 10(a)) and the second using a successive step inputs (Fig. 10(b)). The gains used for these simulations are optimized $\left(\gamma_{1}=5\right.$ and $\left.\gamma_{2}=0.71\right)$ and the two velocity profiles are compared to a path following at a constant speed of $v=100$ pixels/sec. Through the results shown in Table II, it can highlighted that the variation of the velocity during the path following process has no any impact on the quality of the following process. Indeed, the variation of $d$ and $\theta_{e}$ are in the same range with and without velocity variation.

4) Impact of $\Gamma$ Variation during Path Following Process: Not being able to validate the controller on true tissues surface (especially on vocal cords), we simulated a curve $(\Gamma(t))$ whose size changed with a frequency close to that of a patient breathing. This experimentation is shown in Fig. 11
TABLE II: RMS and STD for $d$ (pixels) and $\theta_{e}(\mathrm{rad})$.

\begin{tabular}{|l||c|c||c|c|}
\hline$v$ profile & RMS $(d)$ & STD $(d)$ & RMS $\left(\theta_{e}\right)$ & STD $\left(\theta_{e}\right)$ \\
\hline \hline constant & 0.0086 & 0.0080 & 0.0195 & 0.0195 \\
sinusoidal inputs & 0.0073 & 0.0065 & 0.0174 & 0.0174 \\
steps inputs & 0.0086 & 0.0078 & 0.0198 & 0.0198 \\
\hline
\end{tabular}

which represents a captured image during the path following simulation in a variable curve (see multimedia Extension 1). This test demonstrates that despite the curve variation during the path following achievement, the accuracy remains more than satisfactory i.e., $\operatorname{RMS}(d)=0.0208$ pixels, $\operatorname{STD}(d)$ $=0.0204$ pixels and $\operatorname{RMS}\left(\theta_{e}\right)=0.2447 \mathrm{rad}, \operatorname{STD}\left(\theta_{e}\right)=$ $0.02445 \mathrm{rad}$.

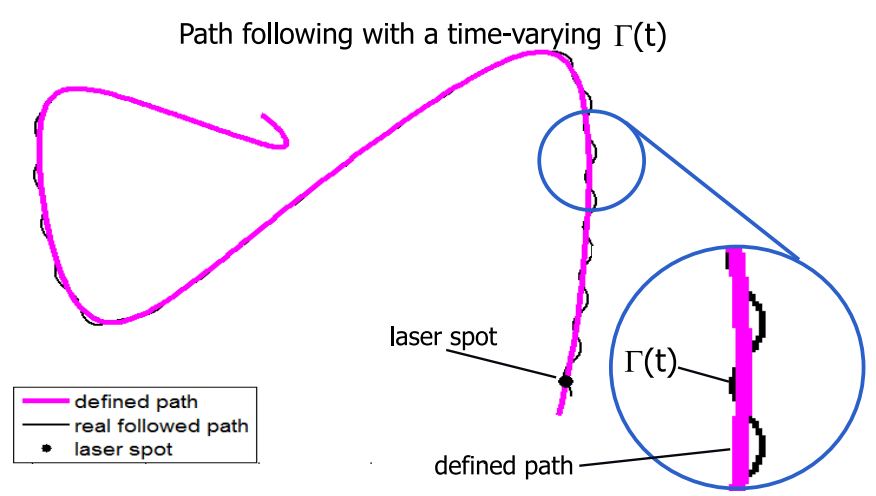

Fig. 11: Image captured during the simulation with a path following with a time-varying curve.

\section{Experimental Validation}

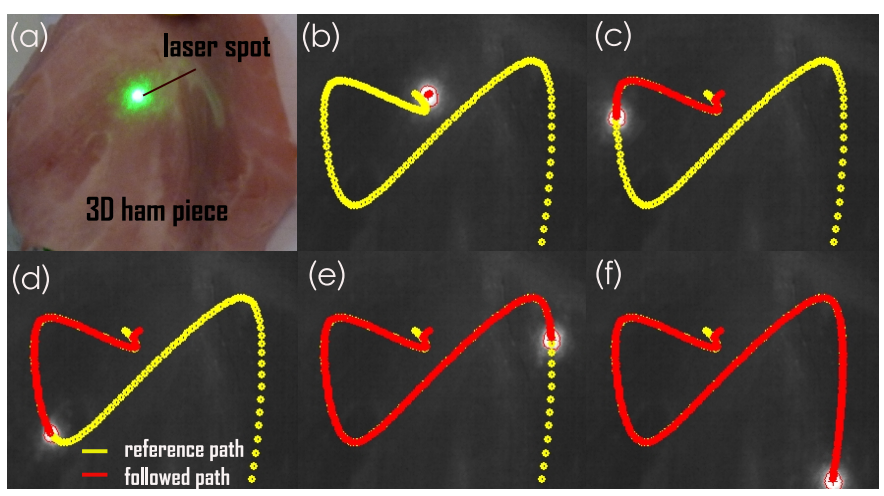

Fig. 12: Image sequence captured during the experimental validation of the path following in the case of unknown shape curve. (a) represents a color image of a 3D ham piece (similar in the texture and the aspect to the vocal folds tissues), and (b) to (f) illustrate the laser spot displacement following the reference path.

The simulation tests have been reproduced on the experimental set-up presented above. Therefore, the path chosen is the arbitrary shape curve used in the simulation section. The initial position of the laser spot is placed at a distance $d=10$ pixels with an initial orientation error $\theta_{e}=0$. This is done again in order to evaluate how the system responds 
to important error ( 10 pixels $=1000$ times the RMS error). To do this, the initial parameters are fixed as follows: $v=20$, $\gamma_{1}=0.2$, and $\gamma_{2}=0.8$. It can be underlined that the initial errors as well as the initial parameters are chosen in a nonoptimal configuration (i.e., huge distance between the laser spot position and the curve $\Gamma$ and non-optimized gains) in order to evaluate the robustness of the controller.

Fig. 12 illustrates an image sequence which shows the achievement of the path following controller in an experimental set-up (see multimedia Extension 1). It can be shown that the laser follows (in red color) perfectly the reference curve (in yellow color) despite the presence of significant curvatures in the curve. Furthermore, Fig. 13(a) gives the evolution of the error distance $d$ and Fig. 13(b) the orientation error $\theta_{e} v s$. the iterations number $k$ (each iteration takes 1.702 milliseconds). Also, It can be noticed that despite the oscillations of $\theta_{e}$, namely in low curvatures areas, this does not affect the quality of the path following i.e., the accuracy (given by the distance error $d$ ) remains high $(\approx 0.22$ pixels $)$.

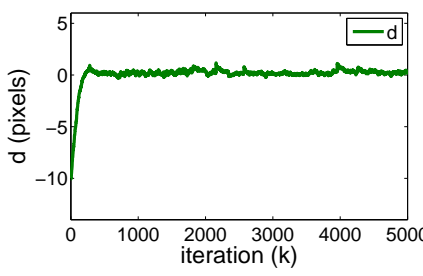

(a) $d$

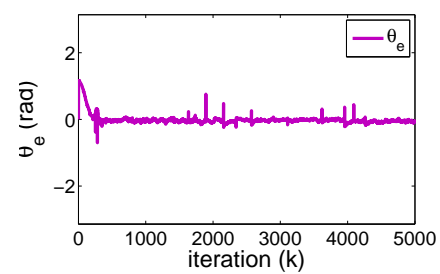

(b) $\theta_{e}$
Fig. 13: Evolution of $d$ and $\theta_{e}$ for the path following validation test shown in Fig. 12

The velocity profile of this test is presented in Fig. 14(a) as well as the velocity of the laser displacement in $\Gamma$ is shown in Fig. 14(b). It demonstrates that the velocity remains constant (with a relative velocity distortion $\frac{\left(v-v_{a c h}\right)}{v}$ below $10^{-6 \%}$ ) between the achieved velocity $v_{a c h}$ and the desired $v$.

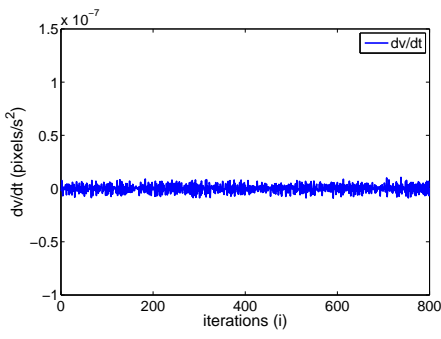

(a) $\frac{\left(v-v_{a c h}\right)}{v}$ (b) $\dot{s}$

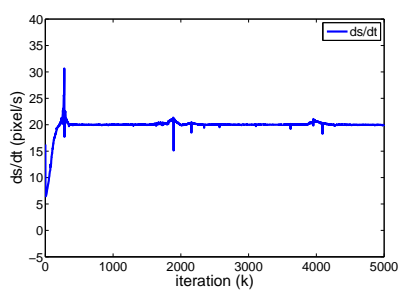

Fig. 14: Velocity profile $\frac{\left(v-v_{a c h}\right)}{v}$ and velocity progress $\dot{s}$ during the validation test shown in Fig. 12

\section{Repeatability of the Path Following Method}

Firstly, for a better assessment of the obtained results, the previous test was reproduced 30 times by changing the initial positions of the laser spot to the curve $\Gamma$ at each test. Thereby, the RMS errors in $d$ and $\theta_{e}$ as well as the STD were computed
TABLE III: RMS and STD for $d$ (pixels) and $\theta_{e}(\mathrm{rad})$. Each line represents the average of 3 individual tests.

\begin{tabular}{|l||c|c||c|c|}
\hline $\mathrm{N}^{\circ}$ & RMS $(d)$ & STD $(d)$ & RMS $\left(\theta_{e}\right)$ & $\operatorname{STD}\left(\theta_{e}\right)$ \\
\hline \hline 1 & 0.230 & 0.556 & 0.217 & 0.556 \\
2 & 0.223 & 0.554 & 0.209 & 0.555 \\
3 & 0.229 & 0.555 & 0.216 & 0.555 \\
4 & 0.224 & 0.555 & 0.210 & 0.555 \\
5 & 0.231 & 0.554 & 0.217 & 0.555 \\
6 & 0.235 & 0.556 & 0.221 & 0.556 \\
7 & 0.225 & 0.554 & 0.211 & 0.554 \\
8 & 0.229 & 0.556 & 0.217 & 0.557 \\
9 & 0.231 & 0.555 & 0.217 & 0.555 \\
10 & 0.234 & 0.555 & 0.220 & 0.555 \\
\hline
\end{tabular}

and reported in Table III. As shown in this Table, the path following is still accurate and repeatable.

Secondly, the experimental validation was reproduced on different curve shapes (e.g.,snake, sinusoidal curve, $\mu R A L P$, unicorn, etc.) using the same initial parameters as in the previous example. It can be shown that the controller remains efficient as illustrated in Table IV.

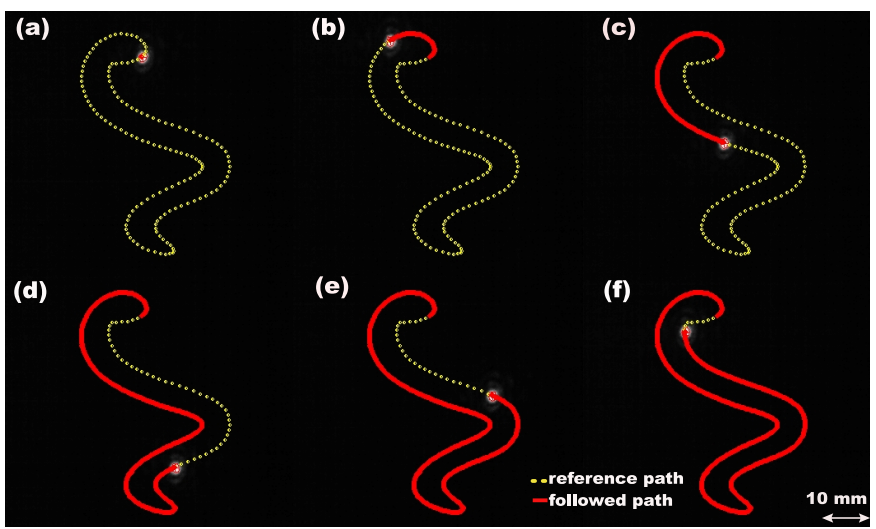

Fig. 15: Image sequence captured during the experimental validation of the path following: (a) to (f) represent the laser spot displacements (in red color) following the reference path (in yellow color).

For instance, Fig. 15 shows an image sequence captured during the achievement of the path following on a snake curve. It can show that the path following is still efficient without any initial parameters optimization. Thus, Fig. 16 presents also a low velocity distortion, despite the complexity of curve, of only $9.1 \times 10^{-9} \%$ with a RMS in $d$ of 0.362 pixels and in $\theta_{e}$ of $0.495 \mathrm{rad}$.

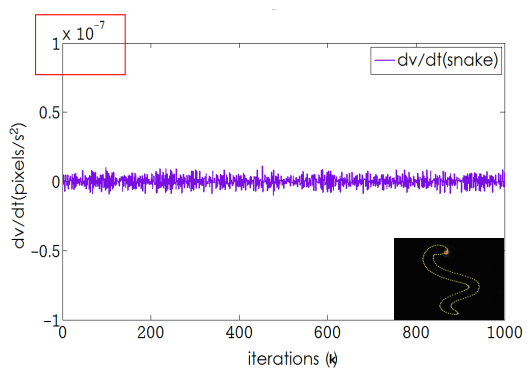

Fig. 16: Velocity profile $\frac{\left(v-v_{a c h}\right)}{v}$ in the snake curve. 
Also, as mentioned above, the controller was evaluated in a more complex curve (i.e., $\mu$ RALP) decomposed into shorten paths: " $\mu$ ", " $r "$, " $a ", " l "$ and " $p "$. As shown in Fig. 17, the path following method is still efficient even with complex and successive curves (see multimedia Extension 1). Again, a low velocity distortion (Fig. 18) which is estimated to $6.9 \times 10^{-7} \%$ and the same order of RMS error in $d$ ( 0.319 pixels $)$ and in $\theta_{e}(0.452 \mathrm{rad})$.

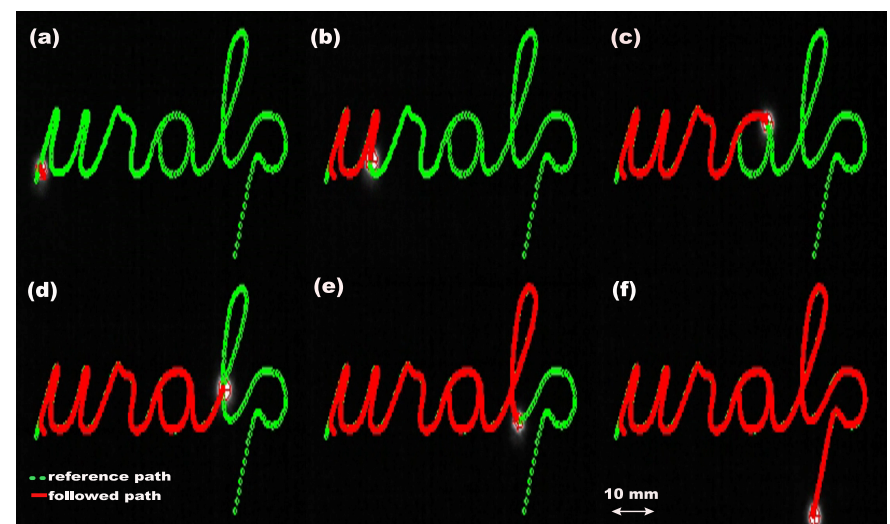

Fig. 17: Image sequence captured during the path following achievement in the $\mu$ RALP: (a) to (f) represent the laser spot displacements (in red color) following the reference path (in green color).

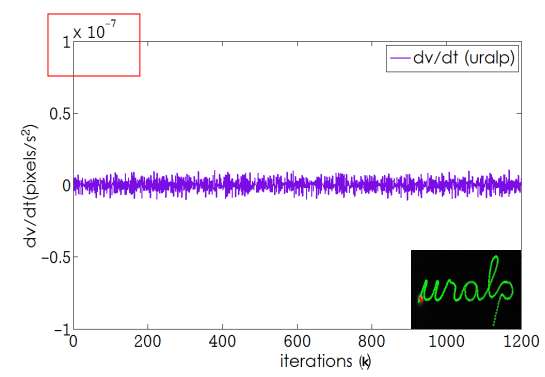

Fig. 18: Velocity profile $\frac{\left(v-v_{a c h}\right)}{v}$ in the $\mu R A L P$ curve.

Finally, Table IV summarizes the RMS errors as well as the STD in $d$ and $\theta_{e}$ of the additional tested curves. Once more, the obtained results are more than satisfactory.

TABLE IV: RMS and STD for $d$ (pixels) and $\theta_{e}(\mathrm{rad})$ for additional paths.

\begin{tabular}{|l||c|c||c|c|}
\hline Type of path & RMS $(d)$ & STD $(d)$ & RMS $\left(\theta_{e}\right)$ & STD $\left(\theta_{e}\right)$ \\
\hline \hline circle & 0.388 & 0.388 & 0.229 & 0.234 \\
sinus & 0.401 & 0.412 & 0.227 & 0.233 \\
$\mu$ RALP & 0.319 & 0.257 & 0.452 & 0.444 \\
unicorn & 0.351 & 0.353 & 0.482 & 0.183 \\
snake & 0.362 & 0.367 & 0.495 & 0.485 \\
\hline
\end{tabular}

\section{E. Using High Values of $v$}

The impact of the velocity $v$ on the quality of the path following is also discussed in this paper. For example, is there a sensitive effect on the RMS and the STD of the error? Thus, the path following algorithm is applied on the unknown shape curve with the same initial parameters (i.e., $\gamma_{1}$ and $\gamma_{2}$ ) but with different velocities $v$ ranging from 100 pixels/second to 500 pixels/second with a step of 100 . From Table V, it can be underlined that the accuracy remains very stable with a RMS ranging from 0.304 to 0.370 pixels for $d$ and from 0.269 to $0.291 \mathrm{rad}$ for $\theta_{e}$, and STD within similar ranges.

TABLE V: RMS and STD for $d$ (pixels) and $\theta_{e}$ ( rad) using different velocities (pixels/second).

\begin{tabular}{|l||c|c||c|c|}
\hline Velocity & RMS $(d)$ & STD $(d)$ & RMS $\left(\theta_{e}\right)$ & STD $\left(\theta_{e}\right)$ \\
\hline \hline 100 & 0.348 & 0.518 & 0.291 & 0.592 \\
200 & 0.323 & 0.521 & 0.283 & 0.625 \\
300 & 0.364 & 0.524 & 0.282 & 0.636 \\
400 & 0.304 & 0.526 & 0.269 & 0.627 \\
500 & 0.370 & 0.527 & 0.271 & 0.639 \\
\hline
\end{tabular}

\section{F. CPU-load of the controller}

Note that in laser microphonosurgery, the laser scanning should works in high frequency i.e., at least of $200 \mathrm{~Hz}$ (sampling time) to avoid tissue carbonization during tumors ablation or resection. As shown in Table VI, it can be noted that our approach takes only 1.702 milliseconds $(\mathrm{ms})$ which is equivalent to a frequency of $587 \mathrm{~Hz}$ (sampling time) despite the use of a slow USB communication.

TABLE VI: CPU-load of the main tasks of the process.

\begin{tabular}{|l|c|}
\hline Task & Time $(\mathrm{ms})$ \\
\hline \hline image grabbing & 0.064 \\
laser tracking & 0.471 \\
controller & 0.002 \\
control sending (USB 2.0) & 0.989 \\
other & 0.176 \\
\hline entire process & $\mathbf{1 . 7 0 2}(\mathbf{f}=\mathbf{5 8 7} \mathbf{H z})$ \\
\hline
\end{tabular}

\section{CONCLUSION}

In this paper, a vision-guided laser steering technique using a path following approach was presented. To do this, we were inspired by the methods used in mobile robotic (unicycle kinematic model) in order to develop an efficient controller scheme by taking into account the fact that the path following and the velocity profile must be decoupled. Thus, the proposed controller was based on the use of the curvilinear representation of the laser steering kinematic model more adapted to our problem.

Our developments were validated in simulation as well as in an experimental set-up, in different conditions of use

- using various initial velocity inputs: step input (fixed laser velocity), sinusoidal inputs, and successive steps inputs;

- starting with large initial errors;

- using low to high laser velocity (from 100 pixels/second to 500 pixels/second;

- using optimized and non-optimized parameters $\gamma_{1}$ and $\gamma_{2}$;

- using time-varying path (i.e., $\Gamma(t))$ simulating a patient breathing;

- using more complex curves with high curvatures (more complex comparing to the laser surgery paths). 
The experimental set-up was equipped with a high frequency $(1 \mathrm{kHz})$ actuated mirror and high speed camera $(\approx$ $10000 \mathrm{fps}$ ). The developed controller has shown more than satisfactory results in terms of accuracy (average accuracy lower than 0.22 pixels $(\approx 10 \mu \mathrm{m})$ with a standard deviation of 0.55 pixels $(\approx 25 \mu \mathrm{m})$, and a relative velocity distortion below $10^{-7} \%$, repeatability (more than 30 repetitions with the same order of accuracy) and frequency $(587 \mathrm{~Hz})$.

The next stages of this work will involve adapting the described materials for real laser surgery applications i.e., on a human cadaver using the entire endoscopic system developed in the $\mu$ RALP project. In addition, the velocity will have to be servoed with regards to the heat profile in the tissue and to the depth variations of the tissues with respect to the image.

\section{ACKNOWLEDGMENT}

This work was supported by $\mu$ RALP, the EC FP7 ICT Collaborative Project no. 288663 (http://www.microralp.eu), and by ACTION, the French ANR Labex no. ANR-11-LABX01-01 (http: //www.labex-action.fr).

\section{REFERENCES}

[1] W. Schwesinger and J. Hunter, "Laser in general surgery," Surgical Clinics of North America, 1992.

[2] V. Moiseev, "Laser technologies in microsurgery of extramedullary tumors," in Int. conf. on Actual Problems of Elect. Inst. Eng., 2004, pp. $275-275$.

[3] H. Eckel, S. Berendes, M. Damm, and J. Klusmann, "Suspension laryngoscopy for endotracheal stenting," Laryngoscope, vol. 113, pp. 11-15, 2003.

[4] M. Remacle, "Laser-assisted microphonosurgery," in Surgery of Larynx and Trachea, Springer Berlin Heidelberg, 2010, pp. 51-56.

[5] L. I. Israel. (2014, Feb.) Digital acublade system. israel@online. [Online]. Available: http://www.surgical.lumenis.com

[6] J. Ortiz, L. Mattos, and D. Caldwell, "Smart devices in robot-assisted laser microsurgery: Towards ubiquitous tele-cooperation," in IEEE Int. Conf.on Rob. and Biomimetics, 2012, pp. S1721-1726.

[7] L. Mattos, D. Nikhil, G. Barresi, L. Gustani, G. Pirreti, " A novel computerized surgeon-machine interface for robot-assisted laser phonomicrosurgery"; in Laryngoscope, DOI: 10.1002/lary.24566, 2014.

[8] T. Sibillano, A. Ancona, V. Berardi, and P. Lugarà, "A real-time spectroscopic sensor for monitoring laser welding processes," Sensors, vol. 9, pp. 3376-3385, 2009.

[9] M. Fridenfalk and G. Bolmsjo, "Design and validation of a universal 6D seam-tracking system in robotic welding using arc sensing," Adv. Rob., vol. 18, pp. 1-21, 2004.

[10] Y. Huang, Y. Xiao, P. Wang, and M. Li, "A seam-tracking laser welding platform with $3 \mathrm{~d}$ and $2 \mathrm{~d}$ visual information fusion vision sensor system," Int. J. of Adv. Manuf. Tech., vol. 67, pp. 415-426, 2012.

[11] H. Cui, Z.Xiao, J. Dong; Y. Chen, G. Hou, Z. Zhao, "Notice of Retraction Analysis on arc-welding robot visual control tracking system," Int. Conf. on Quality, Reliability, Risk, Maint., and Safety Eng., pp. 2091-2094, 2013.

[12] B. Thuilot, R. Lenain, P. Martinet, C. Cariou, "Accurate GPS-based guidance of agricultural vehicles operating on slippery grounds", Chapter 6 in "Focus on Robotics Research", pp. 185-239, John Liu editor, Nova Science Publishers, 2006.

[13] B. Thuilot, J. Bom, F. Marmoiton, P. Martinet, "Accurate automatic guidance of an urban electric vehicle relying on a kinematic GPS sensor", fifth IFAC Symp. on Intel. Auton. Vehi. , Portugal, pp. 1-6, 2004

[14] B. Petrak, K. Konthasinghe, S. Perez, A. Muller, "Feedback-controlled laser fabrication of micromirror substrates," Rev. of Scient. Inst. vol.82(12), pp. 123112-123112-6, 2011

[15] F. Arai, K. Yoshikawa, T. Sakami, T. Fukuda, "Synchronized laser micromanipulation of multiple targets along each trajectory by single laser," Applied Phy. Let. , vol.85(19), pp. 4301-4303, 2004.
[16] R. W. Brockett, "Asymptotic stability and feedback stabilization," in Differential Geometric Control Theory, R.S.M. R.W. Boston: Birkhauser, 1983, pp. 181-191.

[17] A. Bloch, M. Reyhanoglu, and N. McClamroch, "Control and stabilization of nonholonomic dynamic systems," IEEE T. on Aut. Cont., vol. 37 (11), pp. 1746-1757, 1992.

[18] J. P. Hespanha, D. Liberzon, and A. Morse, "Logic-based switching control of a nonholonomic system with parametric modeling uncertainty," Syst. and Cont. Letters, vol. 38(3), pp. 167-177, 1999.

[19] T. Floquet, J.-P. Barbot, and W. Perruquetti, "Higher-order sliding mode stabilization for a class of nonholonomic perturbed systems," Automatica, vol. 39(6), pp. 1077-1083, 2003.

[20] C. Samson, "Velocity and torque feedback control of a nonholonomic cart," in Advanced Rob. Cont., Springer Berlin Heidelberg, 1991, vol. 162, pp. $125-151$

[21] M. Aicardi, G. Casalino, A. Bicchi, and A. Balestrino, "Closed loop steering of unicycle like vehicles via lyapunov techniques," IEEE Rob. Auto. Mag., vol. 2(1), pp. 27-35, 1995.

[22] J. Godhavn and O. Egeland, "A lyapunov approach to exponential stabilization of nonholonomic systems in power form," IEEE T. Auto. Cont., vol. 42(7), pp. 1028-1032, 1997.

[23] C. Samson, "Mobile robot control. part 2: Control of chained systems and application to path following and time-varying point stabilization of wheeled vehicles," INRIA, Tech. Rep. 1994, 1993.

[24] P. Morin and C. Samson, "Motion control of wheeled mobile robots," in Springer Handbook of Robotics, B. Siciliano and O. Khatib, Eds. Springer Berlin Heidelberg, 2008, pp. 799-826.

[25] E. Marchand, F. Spindler, and F. Chaumette, "Visp for visual servoing: a generic software platform with a wide class of robot control skills," IEEE Rob. and Auto. Mag., vol. 12(4), pp. 40-52, 2005.

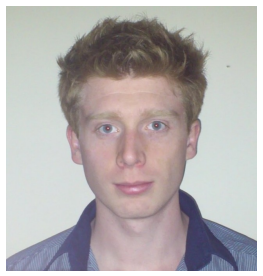

Jean-Antoine Seon holds an Engineer degree in mechanical engineering and micro-technologies from ENSMM (Besançon, France) in 2013 and a Master degree in mechatronics and micro-systems from the Université de Franche-Comté (Besançon, France) in 2014. He is currently a Ph.D student at the AS2M department at the FEMTO-ST Institut where his research area is mainly centered on planning and control for dexterous micro-manipulation.

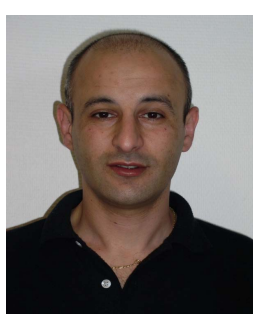

Brahim Tamadazte holds a Ph.D. in Automation and Computer Science from the "Université de Franche-Comté" in 2009 and a MS degree in Robotics and Intelligent Systems from the "Université Pierre et Marie Curie (Paris VI)" in 2005, in France. Since 2012, he joined the FEMTO-ST Institut in the AS2M Departement as Senior Scientist CNRS researcher (Besançon, France). Currently, he works in microrobotics surgery (conception and control) and visual servoing using OCT imaging system.

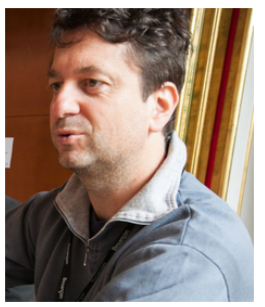

Nicolas Andreff received the Engineer degree (M.S degree) in computer sciences and applied mathematics from ENSEEIHT, Toulouse, France, in 1994, and the Ph.D. degree in computer graphics, computer vision, and robotics from INPG, Grenoble, France, in 1999. He is currently a Professor in the AS2M Department of the Institut FEMTO-ST, Universite de Franche-Comté, Besancon, France. He created the Biomedical Micro-/Nano-Robotics group in the AS2M Department in 2012. His research interests range from vision-based control and parallel kinematics to intracorporeal microrobotics. 